\title{
Dynamo action of the zonal winds in Jupiter
}

\author{
J. Wicht ${ }^{1}$, T. Gastine ${ }^{2}$, L. D. V. Duarte ${ }^{3}$, and W. Dietrich ${ }^{1}$ \\ ${ }^{1}$ Max Planck Institute for Solar System Research, Justus-von-Liebig-Weg 3, 37077 Göttingen, Germany \\ e-mail: wicht@mps.mpg.de \\ ${ }^{2}$ IPGP, Institut de Physique du Globe de Paris, Sorbonne Paris Cité, Université Paris-Diderot, UMR 7154 CNRS, 1 rue Jussieu, \\ 75005 Paris, France \\ ${ }^{3}$ College of Engineering, Mathematics and Physical Sciences, University of Exeter, Physics building, Stocker Road, Exeter, \\ EX4 4QL, UK
}

Received 12 April 2019 / Accepted 5 July 2019

\begin{abstract}
The new data delivered by NASA's Juno spacecraft significantly increase our understanding of the internal dynamics of Jupiter. The gravity data constrain the depth of the zonal flows observed at cloud level and suggest that they slow down considerably at a depth of about $0.96 r_{\mathrm{J}}$, where $r_{\mathrm{J}}$ is the mean radius at the one bar level. The magnetometer onboard Juno reveals the internal magnetic field of the planet. We combine the new zonal flow and magnetic field models with an updated electrical conductivity profile to assess the zonalwind-induced dynamo action, concentrating on the outer part of the molecular hydrogen region of Jupiter where the conductivity increases very rapidly with depth. Dynamo action remains quasi-stationary and can therefore reasonably be estimated where the magnetic Reynolds number remains smaller than one, which is roughly the region above $0.96 r_{\mathrm{J}}$. We calculate that the locally induced radial magnetic field reaches rms values of about $10^{-6} \mathrm{~T}$ in this region and may just be detectable by the Juno mission. Very localized dynamo action and a distinct pattern that reflects the zonal wind system increases the chance to disentangle this locally induced field from the background field. The estimates of the locally induced currents also allow calculation of the zonal-flow-related Ohmic heating and associated entropy production. The respective quantities remain below new revised predictions for the total dissipative heating and total entropy production in Jupiter for any of the explored model combinations. Thus, neither Ohmic heating nor entropy production offer additional constraints on the depth of the zonal winds.
\end{abstract}

Key words. planets and satellites: magnetic fields - planets and satellites: gaseous planets - magnetohydrodynamics (MHD) dynamo

\section{Introduction}

Two of the main objectives of NASA's Juno mission are to measure the magnetic field of Jupiter with unprecedented resolution and to determine the depth of the fierce zonal winds observed in its cloud layer. The first Juno-based internal magnetic field model JRM09 (Connerney et al. 2018) already provides the internal magnetic field up to spherical harmonic degree 10 and shows several interesting features that seem unique to the dynamo of Jupiter (Moore et al. 2018). Better-resolved models are expected as the mission continues.

Based on Juno gravity measurements (Iess et al. 2018), Kaspi et al. (2018) deduce that the speed of the equatorially antisymmetric zonal flow contributions must be significantly reduced at a depth of about $3000 \mathrm{~km}$ below the one-bar level, which corresponds to a radius of $0.96 r_{\mathrm{J}}$. Kong et al. (2018) come to roughly similar conclusions with a different inversion procedure, but they also point out that the solution is not unique. While the gravity data only allow us to constrain the equatorially antisymmetric winds, the results likely also extend to the symmetric contributions. New interior models (Guillot et al. 2018; Debras \& Chabrier 2019) and also the width of the dominant equatorial jet (Gastine et al. 2014; Heimpel et al. 2016) both support the idea that the fast zonal winds are roughly confined to the outer $4 \%$ in radius.

The fast planetary rotation enforces geostrophic flow structures with minimal variation along the direction of the rotation axis. Geostrophic zonal winds are therefore expected to reach right through the gaseous envelope of the planet, and it remains unclear which mechanism limits their extent in Jupiter. The demixing of hydrogen and helium and the subsequent precipitation of helium deeper into the planet offers one possible explanation (Militzer et al. 2016). This process would have established a helium gradient that suppresses convection. In Jupiter, this stable helium-rain layer may start somewhere between 0.93 and $0.90 r_{\mathrm{J}}$ and perhaps extends down to $0.80 r_{\mathrm{J}}$ (Debras \& Chabrier 2019). We note however that ab initio simulations by Schöttler \& Redmer (2018) predict that the hydrogen/helium demixing may not even have started. Recent analysis of gravity measurements by the Cassini spacecraft suggest that the zonal winds of Saturn may only reach down to about $0.85 r_{\mathrm{S}}$ (Iess et al. 2019; Galanti et al. 2019). Since the stably stratified layer is thought to start significantly deeper, at about $0.62 r_{\mathrm{S}}$ according to Schöttler \& Redmer (2018), it cannot be the reason for the limited depth of the zonal winds.

A second possible mechanism braking of the zonal winds at depth involves the Lorentz forces. Lorentz forces are tied to dynamo action and thus to the electrical conductivity profile. Ab initio simulations for Jupiter suggest that ionization effects lead to a super-exponential increase of the electrical conductivity in the outermost molecular gas envelope. We refer to this layer as the Steeply Decaying Conductivity Region (SDCR) of Jupiter in the following. At about $0.9 r_{\mathrm{J}}$, hydrogen, the main constituent of the planet, becomes metallic, and the conductivity increases 


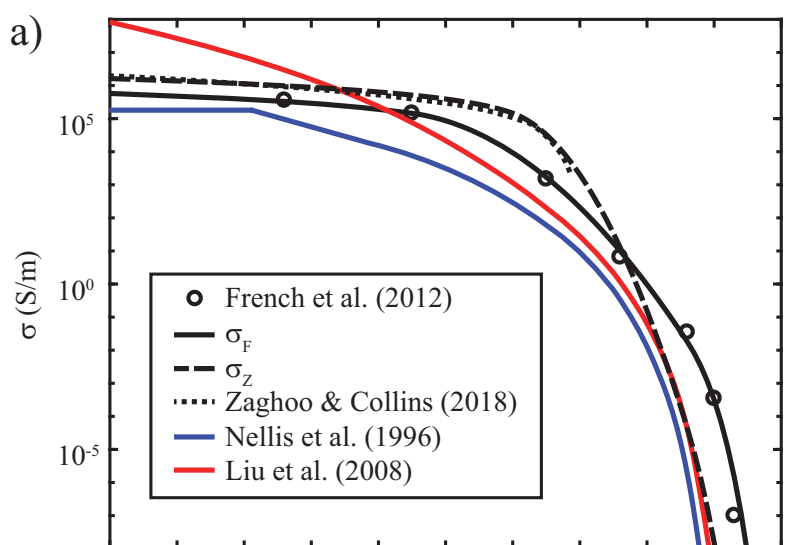

$\begin{array}{lllllllllll}0.80 & 0.82 & 0.84 & 0.86 & 0.88 & 0.90 & 0.92 & 0.94 & 0.96 & 0.98 & 1.00\end{array}$ $\mathrm{r} / \mathrm{r}_{\mathrm{J}}$

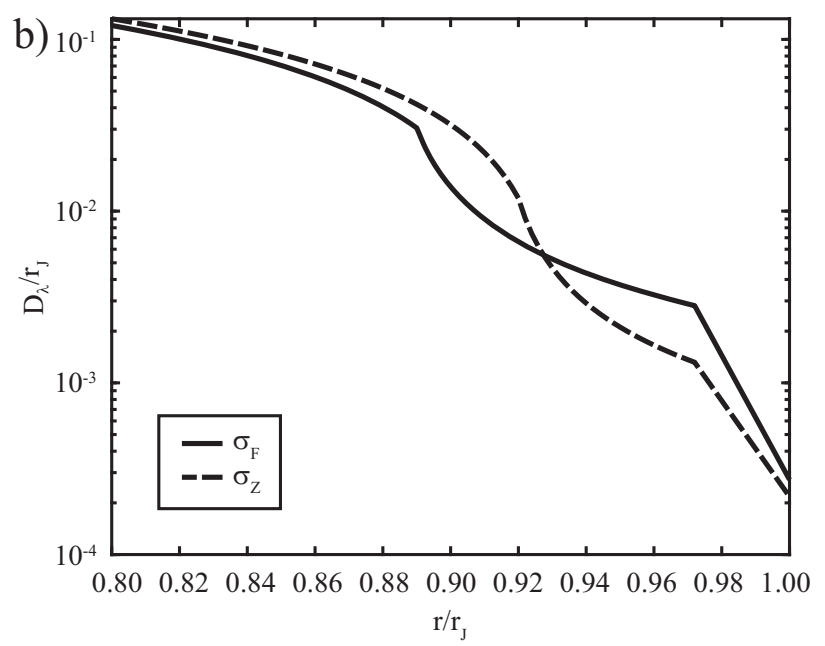

Fig. 1. Panel $a$ : electrical conductivity profiles in the outer $20 \%$ of the radius of Jupiter. The black line shows the parametrization $\sigma_{F}(r)$ of the ab initio simulation data points (black circles) by French et al. (2012). The dotted red line shows the profile published in Zaghoo \& Collins (2018), while the solid red line shows the extension $\sigma_{Z}(r)$ used here. The profiles suggested by Liu et al. (2008) (green) and Nellis et al. (1999) (blue) are shown for comparison. Panel $b$ : magnetic diffusivity scale height $D_{\lambda} / r_{\mathrm{J}}$ for $\sigma_{F}$ and $\sigma_{Z}$.

much more smoothly with depth (French et al. 2012); see panel a of Fig. 1. Though dynamo action and the potential braking of the zonal winds due to Lorentz forces are classically attributed to the metallic region, they may already become significant where the electrical conductivity reaches sizable levels in the SDCR of Jupiter.

Different dynamo-related arguments have been evoked to estimate the depth of the zonal winds without however directly addressing the role of the Lorentz forces. Liu et al. (2008) estimate that the Ohmic heating caused by zonal-wind-related induction would exceed the total heat emitted from Jupiter's interior should the winds reach deeper than $0.96 r_{\mathrm{J}}$ with undiminished speed. Ridley \& Holme (2016) argue that the secular variation of the magnetic field over $30 \mathrm{yr}$ of pre-Juno observations is relatively small and is thus likely incompatible with an advection by undiminished zonal winds. These latter authors conclude that the winds cannot reach depths where the magnetic Reynolds number exceeds one and more significant induction can be expected. This puts the maximum depth somewhere between $0.96 r_{\mathrm{J}}$ and $0.97 r_{\mathrm{J}}$, as we discuss below. A recent analysis by Moore et al. (2019) suggests that the observations over a $45 \mathrm{yr}$ time-span, including Juno data, would be compatible with zonal wind velocities of $2.4 \mathrm{~m} / \mathrm{s}$ at $0.95 r_{\mathrm{J}}$, two orders of magnitude lower than observed in the cloud layer.

Another interesting question is how much the dynamo action in the SDCR of Jupiter contributes to the total magnetic field. Using a simplified mean-field approach, Cao \& Stevenson (2017) predict that the radial component of the locally induced field (LIF) may reach $1 \%$ of the background field and could therefore be detectable by the Juno magnetometer. Wicht et al. (2019) analyze the dynamo action in the SDCR of fully self-consistent numerical simulations that yield Jupiter-like magnetic fields. Because of the dominance of Ohmic diffusion, the dynamo dynamics becomes quasi-stationary in the SDCR of their simulations. A consequence is that the locally induced electric currents and field can be estimated with relatively good precision when flow, electrical conductivity profile, and the surface magnetic field are known. The new constraints on the internal magnetic field of Jupiter and the depth of the zonal winds provided by the Juno mission, combined with improved estimates on the electrical conductivity profile, allow a fresh look at the problem.

Here we use three different zonal flow models, two electrical conductivity models, and the new Juno-based magnetic field model JRM09 to predict the electric currents and magnetic fields produced in the SDCR of Jupiter. In addition, we also derive new estimates for the total dissipative heating and related entropy production and explore whether either value is exceeded by the zonal-flow-related Ohmic dissipation.

Section 2 outlines the methods and introduces the data we used. Section 3 discusses dissipative heating and entropy production in Jupiter. Estimates for dynamo action, Ohmic heating, and entropy production are then presented in Sect. 4. Section 5 closes the article with a discussion and conclusion.

\section{Methods and data}

\subsection{Estimating dynamo action}

The ratio of inductive to diffusive effects in the induction equation,

$$
\frac{\partial \boldsymbol{B}}{\partial t}=\boldsymbol{\nabla} \times(\boldsymbol{U} \times \boldsymbol{B})-\boldsymbol{\nabla} \times \lambda \boldsymbol{\nabla} \times \boldsymbol{B},
$$

can be quantified by the magnetic Reynolds number

$R m=\frac{\langle\boldsymbol{U}\rangle D}{\lambda}$,

where $\lambda=1 /(\mu \sigma)$ is the magnetic diffusivity, with $\mu$ the magnetic permeability and $\sigma$ the electrical conductivity. Angular brackets generally denote rms values at a given radius throughout the paper; thus $\langle\boldsymbol{U}\rangle$ stands for

$\langle\boldsymbol{U}\rangle=\left(\frac{1}{4 \pi} \int_{0}^{2 \pi} \mathrm{d} \phi \int_{0}^{\pi} \mathrm{d} \theta \sin \theta \boldsymbol{U}^{2}\right)^{1 / 2}$,

with $\theta$ being the colatitude and $\phi$ the longitude.

The typical length scale $D$ is hard to estimate, and the planetary radius is often used for simplicity. Since $\sigma$ decreases steeply in the SDCR, the length scale is determined by the conductivity or magnetic diffusivity scale height:

$D_{\lambda}=\frac{\lambda}{\partial \lambda / \partial r}=-\frac{\sigma}{\partial \sigma / \partial r}$ 
and the modified magnetic Reynolds number,

$R m^{(1)}=\frac{\langle\boldsymbol{U}\rangle D_{\lambda}}{\lambda}$

should be used. Since $D_{\lambda}$ is small and $\lambda$ decreases steeply with radius, most of the SDCR of Jupiter is characterized by a small magnetic Reynolds number $R m^{(1)}<1$, and the magnetic field dynamics becomes quasi-stationary (Liu et al. 2008), obeying the simplified induction equation

$\boldsymbol{\nabla} \times \frac{\boldsymbol{j}}{\sigma} \approx \boldsymbol{\nabla} \times(\boldsymbol{U} \times \tilde{\boldsymbol{B}})$

Here, $\boldsymbol{j}$ is the current density and $\tilde{\boldsymbol{B}}$ the strong background field produced by the dynamo acting deeper in the planet. The LIF $\hat{\boldsymbol{B}}$ is given by Ampere's law:

$\boldsymbol{j}=\boldsymbol{\nabla} \times \hat{\boldsymbol{B}} / \mu$.

The steep $\sigma$ profile dominates the radial dependence of $\boldsymbol{j}$ and $\hat{\boldsymbol{B}}$ in the SDCR. The current density is thus dominated by the horizontal components, where radial gradients in $\hat{\boldsymbol{B}}$ contribute (Liu et al. 2008; Wicht et al. 2018):

$\boldsymbol{j} \approx \boldsymbol{j}_{H} \approx \hat{\mathbf{r}} \times \frac{\partial}{\partial r} \hat{\boldsymbol{B}}_{H}$

Index $H$ denotes the horizontal components; the radial current can be neglected in comparison.

Along the same lines, the horizontal components of Eq. (6) can be approximated by

$\frac{1}{r} \frac{\partial}{\partial r} \frac{r}{\sigma} \boldsymbol{j}_{H} \approx-\hat{\mathbf{r}} \times[\boldsymbol{\nabla} \times(\boldsymbol{U} \times \tilde{\boldsymbol{B}})]_{H}$,

where $\hat{\mathbf{r}}$ is the radial unit vector. Integration in radius yields the integral current density estimate introduced by Liu et al. (2008), which we identify with an upper index $(I)$ :

$\boldsymbol{j}_{H}^{(I)}=\frac{\sigma}{r}\left[\frac{r}{\sigma} \boldsymbol{j}_{H}\right]_{r_{J}}+\hat{\mathbf{r}} \times \frac{\sigma}{r} \int_{r}^{r_{J}} \mathrm{~d} r^{\prime} r^{\prime}[\boldsymbol{\nabla} \times(\boldsymbol{U} \times \tilde{\boldsymbol{B}})]_{H}$.

The square brackets with a lower index $r_{\mathrm{J}}$ indicate that the expression should be evaluated at the outer boundary.

For a predominantly zonal flow, we can use the approximation

$$
\begin{aligned}
{[\boldsymbol{\nabla} \times(\boldsymbol{U} \times \tilde{\boldsymbol{B}})]_{H} \approx } & -\frac{\bar{U}_{\phi}}{r \sin \theta}\left(\frac{\partial}{\partial \phi} \tilde{B}_{\theta}\right) \hat{\theta} \\
& +\frac{1}{r}\left[\frac{\partial}{\partial r}\left(r \bar{U}_{\phi} \tilde{B}_{r}\right)+\frac{\partial}{\partial \theta}\left(\bar{U}_{\phi} \tilde{B}_{\theta}\right)\right] \hat{\phi}
\end{aligned}
$$

where $\bar{U}_{\phi}$ is the zonal flow component and $\hat{\theta}$ and $\hat{\phi}$ are unit vectors in latitudinal and azimuthal direction, respectively.

The integral estimates for the two horizontal current components are then given by

$$
\begin{aligned}
j_{\theta}^{(I)}= & \frac{\sigma}{r}\left[\frac{r}{\sigma} j_{\theta}\right]_{r_{J}}-\frac{\sigma}{r}\left(\left[r \bar{U}_{\phi} \tilde{B}_{r}\right]_{r_{J}}-\left[r \bar{U}_{\phi} \tilde{B}_{r}\right]_{r}\right) \\
& -\frac{\sigma}{r} \int_{r}^{r_{J}} \mathrm{~d} r^{\prime} \frac{\partial}{\partial \theta}\left(\bar{U}_{\phi} \tilde{B}_{\theta}\right),
\end{aligned}
$$

and

$j_{\phi}^{(I)}=\frac{\sigma}{r}\left[\frac{r}{\sigma} j_{\phi}\right]_{r_{J}}-\frac{\sigma}{r} \int_{r}^{r_{J}} \mathrm{~d} r^{\prime} \frac{\bar{U}_{\phi}}{\sin \theta} \frac{\partial}{\partial \phi} \tilde{B}_{\theta}$.

Since the latitudinal length scale of the zonal winds is smaller than the azimuthal length scale of the magnetic field, we expect that the latitudinal component dominates.

The integral estimate requires the knowledge of the surface currents. While the surface currents are certainly very small, the scaled version $\sigma(r) / \sigma\left(r_{J}\right) \boldsymbol{j}$ may remain significant. Liu et al. (2008) argue that neglecting the surface contribution at least provides a lower bound for the rms current density.

Wicht et al. (2019) confirm that the dynamics indeed becomes quasi-stationary in the SDCR of their Jupiter-like dynamo simulations and that $j_{\theta}$ is indeed the dominant current component where $\mathrm{Rm}^{(1)}<1$. They also report that the simplified Ohm's law for a fast-moving conductor,

$\boldsymbol{j}^{(O)}=\sigma(\boldsymbol{U} \times \tilde{\boldsymbol{B}})$,

provides a significantly better estimate than $\boldsymbol{j}^{(I)}$. We identify the respective current estimate with an upper index $(O)$. The general Ohm law,

$\boldsymbol{j}=\sigma(\boldsymbol{U} \times \boldsymbol{B}+\mathbf{E})$,

also contains currents driven by the electric field, which reduces to $\mathbf{E}=-\nabla \Phi$ in the quasi-stationary case, where $\Phi$ is the electric potential. In the SDCR of Jupiter, this contribution likely proves secondary because the potential differences remain small compared to the induction by fast zonal winds (Wicht et al. 2019).

As the electrical conductivity decreases in the SDCR, the magnetic field approaches a potential field with its characteristic radial dependence. We use this dependence to approximate the background field with

$\tilde{\boldsymbol{B}}_{\ell}(r, \theta, \phi) \approx\left(\frac{r_{J}}{r}\right)^{\ell+2} \boldsymbol{B}_{\ell}\left(r_{J}, \theta, \phi\right)$,

where the index $\ell$ denotes the magnetic field contribution at spherical harmonic degree $\ell$. This provides a suitable approximation as long as the LIF remains a small contribution of the total field (Wicht et al. 2019).

Given a surface field model and an electrical conductivity profile, Ohm's law for a fast moving conductor and a predominantly zonal flow suggests

$\boldsymbol{j} \approx j_{\theta}^{(O)} \hat{\theta}=\sigma \bar{U}_{\phi} \tilde{B}_{r} \hat{\theta}$.

When using this result to constrain the outer-boundary currents, the alternative integral estimates, Eqs. (12) and (13), yield

$j_{\theta}^{(I)}=\sigma \bar{U}_{\phi} \tilde{B}_{r}-\frac{\sigma}{r} \int_{r}^{r_{J}} \mathrm{~d} r^{\prime} \frac{\partial}{\partial \theta}\left(\bar{U}_{\phi} \tilde{B}_{\theta}\right)$

and

$j_{\phi}^{(I)}=-\frac{\sigma}{r} \int_{r}^{r_{J}} \mathrm{~d} r^{\prime} \frac{\bar{U}_{\phi}}{\sin \theta} \frac{\partial}{\partial \phi} \tilde{B}_{\theta}$,

respectively. A comparison of the estimates shows that $\boldsymbol{j}^{(I)}$ and $j^{(O)}$ will remain very similar at shallow depths. When the flow decays very deeply with depth however, the integral contributions in Eqs. (18) and (19) dominate below some radius and cause larger deviations, as we show below.

Calculating the LIF requires to uncurl Ampere's law, which reduces to integrating Eq. (8) in the SDCR of Jupiter. When 
using $\boldsymbol{j}^{(O)}$, this yields

$\hat{\boldsymbol{B}}_{H} \approx \int_{r}^{r_{J}} \mathrm{~d} r^{\prime} \frac{\hat{\mathbf{r}} \times(\boldsymbol{U} \times \tilde{\boldsymbol{B}})}{\lambda}$.

Since the electrical conductivity profile rules the radial dependence, the integral can be approximated by

$\hat{\boldsymbol{B}}_{H} \approx \frac{D_{\lambda}}{\lambda} \hat{\mathbf{r}} \times(\boldsymbol{U} \times \tilde{\boldsymbol{B}})$

We assume here that the LIF vanishes at the outer boundary. For a dominantly azimuthal flow, the primary LIF component is also azimuthal:

$\hat{B}_{\phi} \approx \frac{D_{\lambda}}{\lambda} \bar{U}_{\phi} \tilde{B}_{r}$

This suggests that the rms value scales with $R m^{(1)}$,

$\left\langle\hat{\boldsymbol{B}}_{H}\right\rangle \approx \operatorname{Rm}^{(1)}\langle\tilde{\boldsymbol{B}}\rangle$,

assuming that the correlation between $\bar{U}_{\phi}$ and $\tilde{B}_{r}$ is of little relevance.

The radial LIF can be estimated based on the radial component on the quasi-stationary induction Eq. (1):

$\lambda \nabla^{2} \hat{B}_{r} \approx-\hat{\mathbf{r}} \cdot \nabla \times(\boldsymbol{U} \times \tilde{\boldsymbol{B}})$.

When approximating Ohmic dissipation by $\lambda \hat{B}_{r} / D_{\lambda}^{2}$, this yields:

$\hat{B}_{r} \approx-\frac{D_{\lambda}^{2}}{\lambda} \hat{\mathbf{r}} \cdot \boldsymbol{\nabla} \times(\boldsymbol{U} \times \tilde{\boldsymbol{B}})$,

which reduces to

$\hat{B}_{r} \approx-\frac{D_{\lambda}^{2}}{\lambda} \frac{\bar{U}_{\phi}}{r \sin \theta} \frac{\partial}{\partial \phi} \tilde{B}_{r}$

for a predominantly zonal flow. This suggest that the rms radial LIF should roughly scale with the second modified magnetic Reynolds number

$R m^{(2)}=\frac{\langle\boldsymbol{U}\rangle D_{\lambda}^{2}}{\lambda D}$

like

$\left\langle\hat{B}_{r}\right\rangle \approx R m^{(2)} \frac{D}{D_{\phi}}\langle\tilde{B}\rangle$

Here, $D_{\phi}$ is the azimuthal length scale of the background field. Since $D_{\lambda} \ll D_{\phi}$, the radial LIF is much smaller than its horizontal counterpart (Wicht et al. 2019).

\subsection{Data}

The electric current and LIF estimates discussed above require a conductivity profile, a zonal flow model, and a surface magnetic field model. For the heating and entropy estimates that we derived in Sect. 3, we also need density, temperature, and thermal conductivity profiles. We adopt the interior model calculated by Nettelmann et al. (2012) and French et al. (2012), which is the only one that provides all the required information. We note however that recent Juno gravity data suggest that the interior of Jupiter may be more complex than anticipated in this model (Debras \& Chabrier 2019).

$\mathrm{Ab}$ initio simulations of the electrical conductivity by French et al. (2012) provide 12 data points at different depths. Figure 1 shows the values in the outer $20 \%$ of the radius of Jupiter and the parametrization $\sigma_{F}(r)$ developed for our analysis. A linear branch,

$\sigma_{F}(r)=\sigma_{r}+\left(\sigma_{m}-\sigma_{r}\right) \frac{r-r_{r}}{r_{m}-r_{r}}$

covers the smoother inner part $r<r_{m}$. An exponential branch,

$\sigma_{F}(r)=\sigma_{m} \exp \left(\left[\frac{r-r_{m}}{r_{m}-r_{r}}+b\left(\frac{r-r_{m}}{r_{m}-r_{r}}\right)^{2}\right] \frac{\sigma_{m}-\sigma_{r}}{\sigma_{m}}\right)$,

describes the steeper decay for $r_{m}<r<r_{e}$ with $b=7.2$. Matching radius $r_{m}=0.89 r_{\mathrm{J}}$ and reference radius $r_{r}=0.77 r_{\mathrm{J}}$ are chosen where ab initio data points have been provided.

A double-exponential branch,

$\sigma_{F}(r)=\sigma_{e} \exp \left(d\left[\exp \left(c \frac{r-r_{e}}{r_{m}-r_{r}}\right)-1\right]\right)$,

is required to capture the super-exponential decrease for $r \geq r_{e}=$ $0.972 r_{\mathrm{J}}$. The additional free parameter is $c=10$, while $\sigma_{e}=$ $\sigma\left(r_{e}\right)$ and

$d=\frac{1}{c}\left(1+2 b \frac{r_{e}-r_{m}}{r_{m}-r_{i}}\right) \frac{\sigma_{m}-\sigma_{r}}{\sigma_{m}}$.

The dotted red line in panel a of Fig. 1 shows the conductivity model used to study dynamo action in Jupiter and Jupiter-like exoplanets by Zaghoo \& Collins (2018). This is based on measurements which suggest a higher electrical conductivity in the metallic hydrogen phase than previous data. Unfortunately, Zaghoo \& Collins (2018) do not discuss how the results were extrapolated to Jovian conditions. The solid red line in Fig. 1 shows the respective parametrization $\sigma_{Z}(r)$ used for our analysis, which retraces the published curve and connects to previously published parametrizations (green and blue) at lower densities (Nellis et al. 1996; Liu et al. 2008). We note however that these parametrizations are based on data (Weir et al. 1996) which may have been attributed to overly low temperatures according to a recent analysis by Knudson et al. (2018).

Though model $\sigma_{Z}(r)$ is somewhat arbitrary, it serves to illustrate the impact of conductivity uncertainties in our study. Close to $r_{\mathrm{J}}$ where conductivities remain insignificant, $\sigma_{F}$ is many orders of magnitude larger than $\sigma_{Z}$. The ratio $\sigma_{F} / \sigma_{Z}$ decreases with depth, reaching $10^{2}$ around $0.97 r_{\mathrm{J}}$ and 10 around $0.96 r_{\mathrm{J}}$. The two models finally cross at about $0.95 r_{\mathrm{J}}$. At about $0.925 r_{\mathrm{J}}$, the ratio reaches a minimum of 0.05 and then slowly increases with depth to 0.35 at $0.8 r_{\mathrm{J}}$. Table 1 list values of both conductivity models for selected radii.

Panel b of Fig. 1 and selected values listed in Table 1 demonstrate that the magnetic diffusivity scale heights $D_{\lambda}$ differ much less than the conductivities themselves. Electric currents, LIFs, and Ohmic heating depend linearly on $\sigma$ but on different powers of $D_{\lambda}$. The differences between the results for the two conductivity models is therefore predominantly determined by $\sigma$ and can easily be scaled from one to the other.

The three different zonal flow models explored here are illustrated in Fig. 2. Table 1 lists rms values $\left\langle\bar{U}_{\phi}\right\rangle$ at selected radii. All reproduce the observed zonal winds at $r=r_{\mathrm{J}}$ (Porco et al. 2003; 
Table 1. Rms flow velocities, electrical conductivities $\sigma$, magnetic diffusivities $\lambda$, diffusivity scale heights $D_{\lambda}$, and magnetic Reynolds numbers $R m^{(1)}$ at selected radii.

\begin{tabular}{cccccccc}
\hline \hline$r / r_{\mathrm{J}}$ & 0.98 & 0.97 & 0.96 & 0.95 & 0.94 & 0.92 & 0.90 \\
\hline$\left\langle U_{G}\right\rangle\left(\mathrm{m} \mathrm{s}^{-1}\right)$ & $3.0 \times 10^{1}$ & $3.2 \times 10^{1}$ & $3.5 \times 10^{1}$ & $3.8 \times 10^{1}$ & $4.1 \times 10^{1}$ & $2.5 \times 10^{1}$ & $1.5 \times 10^{1}$ \\
$\left\langle U_{K}\right\rangle\left(\mathrm{m} \mathrm{s}^{-1}\right)$ & $2.0 \times 10^{1}$ & $1.4 \times 10^{1}$ & $8.0 \times 10^{0}$ & $4.0 \times 10^{0}$ & $2.0 \times 10^{0}$ & $2.5 \times 10^{-1}$ & $4.0 \times 10^{-2}$ \\
$\left\langle U_{Z}\right\rangle\left(\mathrm{m} \mathrm{s}^{-1}\right)$ & $2.3 \times 10^{1}$ & $1.9 \times 10^{1}$ & $1.5 \times 10^{1}$ & $1.1 \times 10^{1}$ & $6.8 \times 10^{0}$ & $1.7 \times 10^{0}$ & $2.4 \times 10^{-1}$ \\
\hline$\sigma_{F}\left(\mathrm{~S} \mathrm{~m}^{-1}\right)$ & $3.2 \times 10^{-4}$ & $3.7 \times 10^{-2}$ & $9.7 \times 10^{-1}$ & $1.7 \times 10^{1}$ & $2.1 \times 10^{2}$ & $9.3 \times 10^{3}$ & $8.6 \times 10^{4}$ \\
$\sigma_{Z}\left(\mathrm{~S} \mathrm{~m}^{-1}\right)$ & $1.5 \times 10^{-8}$ & $1.9 \times 10^{-4}$ & $1.6 \times 10^{-1}$ & $3.4 \times 10^{1}$ & $2.0 \times 10^{3}$ & $1.5 \times 10^{5}$ & $3.9 \times 10^{5}$ \\
$\lambda_{F}\left(\mathrm{~m} \mathrm{~s}^{-2}\right)$ & $2.5 \times 10^{9}$ & $2.2 \times 10^{7}$ & $8.2 \times 10^{5}$ & $4.6 \times 10^{4}$ & $3.8 \times 10^{3}$ & $8.5 \times 10^{1}$ & $9.2 \times 10^{0}$ \\
$\lambda_{Z}\left(\mathrm{~m} \mathrm{~s}^{-2}\right)$ & $5.2 \times 10^{13}$ & $4.1 \times 10^{9}$ & $5.1 \times 10^{6}$ & $2.3 \times 10^{4}$ & $3.9 \times 10^{2}$ & $5.5 \times 10^{0}$ & $2.0 \times 10^{0}$ \\
$D_{\lambda}\left(\sigma_{F}\right) / r_{J}$ & $1.4 \times 10^{-3}$ & $2.9 \times 10^{-3}$ & $3.2 \times 10^{-3}$ & $3.7 \times 10^{-3}$ & $4.4 \times 10^{-3}$ & $6.6 \times 10^{-3}$ & $1.4 \times 10^{-2}$ \\
$D_{\lambda}\left(\sigma_{Z}\right) / r_{\mathrm{J}}$ & $7.9 \times 10^{-4}$ & $1.4 \times 10^{-3}$ & $1.7 \times 10^{-3}$ & $2.1 \times 10^{-3}$ & $2.9 \times 10^{-3}$ & $1.2 \times 10^{-2}$ & $3.3 \times 10^{-1}$ \\
\hline$R m^{(1)}\left(U_{G}, \sigma_{F}\right)$ & $1.2 \times 10^{-3}$ & $2.9 \times 10^{-1}$ & $9.6 \times 10^{0}$ & $2.1 \times 10^{2}$ & $3.2 \times 10^{3}$ & $1.4 \times 10^{5}$ & $1.5 \times 10^{6}$ \\
$R m^{(1)}\left(U_{K}, \sigma_{F}\right)$ & $8.2 \times 10^{-4}$ & $1.3 \times 10^{-1}$ & $2.2 \times 10^{0}$ & $2.3 \times 10^{1}$ & $1.6 \times 10^{2}$ & $1.4 \times 10^{3}$ & $4.2 \times 10^{3}$ \\
$R m^{(1)}\left(U_{Z}, \sigma_{F}\right)$ & $9.1 \times 10^{-4}$ & $1.8 \times 10^{-1}$ & $4.2 \times 10^{0}$ & $6.2 \times 10^{1}$ & $5.4 \times 10^{2}$ & $9.3 \times 10^{3}$ & $2.6 \times 10^{4}$ \\
$R m^{(1)}\left(U_{G}, \sigma_{F}\right)$ & $3.2 \times 10^{-8}$ & $7.4 \times 10^{-4}$ & $7.9 \times 10^{-1}$ & $2.4 \times 10^{2}$ & $2.1 \times 10^{4}$ & $3.8 \times 10^{6}$ & $1.6 \times 10^{7}$ \\
$R m^{(1)}\left(U_{K}, \sigma_{F}\right)$ & $2.2 \times 10^{-8}$ & $3.2 \times 10^{-4}$ & $1.8 \times 10^{-1}$ & $2.5 \times 10^{1}$ & $1.0 \times 10^{3}$ & $3.9 \times 10^{4}$ & $4.3 \times 10^{4}$ \\
$R m^{(1)}\left(U_{Z}, \sigma_{F}\right)$ & $2.4 \times 10^{-8}$ & $4.5 \times 10^{-4}$ & $3.4 \times 10^{-1}$ & $6.9 \times 10^{1}$ & $3.5 \times 10^{3}$ & $2.7 \times 10^{5}$ & $2.7 \times 10^{5}$ \\
\hline
\end{tabular}

Vasavada \& Showman 2005). We use a running average of the surface profile with a window width of one degree and represent the result with 256 (nearly) evenly spaced latitudinal grid points for our calculations.

The three flow models differ at depth. The most simple one, $U_{G}$, assumes geostrophy in each hemisphere, that is, the flow depends only on the distance $s=r \sin \theta$ to the rotation axis. Kaspi et al. (2018) describe the depth decay of the equatorially antisymmetric zonal flow with profiles constrained by the Juno gravity measurements. We apply their "latitude-independent" model version to the total zonal flow and refer to this model as $U_{K}$. The rms amplitude of $U_{K}$ has decreased by one order of magnitude at about $0.95 r_{\mathrm{J}}$ and by two orders of magnitude around $0.925 r_{\mathrm{J}}$.

We also consider the "deep" model suggested by Kong et al. (2018), who assume an exponential depth decay and an additional linear dependence on the distance $z=\cos \theta$ to the equatorial plane. Like for $U_{K}$, our respective model $U_{Z}$ assumes that the depth and $z$ dependences, which were originally derived for the equatorially antisymmetric contributions, apply to the whole flow. The rms velocity in $U_{Z}$ decays more smoothly with depth than in $U_{K}$, having decreased by one order of magnitude at about $0.935 r_{\mathrm{J}}$ and by two orders of magnitude at about $0.905 r_{\mathrm{J}}$.

Figure 2 shows that $U_{G}$ and $U_{K}$ have discontinuities at the equatorial plane. These pose a problem when calculating the latitudinal zonal flow derivatives required for the integral estimate $j_{\theta}^{(I)}$ (see Eq. (18)). Formally, the derivative becomes infinite at the equator. Practically however, the impact of the discontinuity depends on the model setup and on the methods used for calculating the derivatives. We tested the impact on rms current density estimates by comparing calculations covering all latitudes with counterparts where the derivatives were explicitly set to zero in a six-degree belt around the equator. Simple first-order finite differences with 256 grid points at each radial level are generally used for calculating the derivative. For flow $U_{Z}$, which has been constructed to avoid the discontinuity (Kong et al. 2018), the belt contributes not more than one percent to $\left\langle j_{\theta}^{(I)}\right\rangle$ at any radius, which is less than the surface fraction it represents. For flow $U_{K}$, the contribution is even smaller due to the faster decay of the flow amplitude. However, for $U_{G}$ the belt contributes $20 \%$ to the rms current for radii below $0.94 r_{\mathrm{J}}$, which is a clear sign that the unphysical discontinuity causes problems. We take a conservative approach and only consider flow model $U_{Z}$ in connection with estimate $j_{\theta}^{(I)}$ below.

The radius where $R m^{(1)}=1$, which we refer to as $r_{1}$ in the following, roughly marks the point where the approximations discussed above break down (Wicht et al. 2019). Figure 3a illustrates the $R m^{(1)}$ profiles that result from combining $\sigma_{F}$ and $\sigma_{Z}$ with rms values for the three zonal flow models. Table 1 compares values at some selected radii. These modified magnetic Reynolds numbers exceed unity between $r_{1}=0.957 r_{\mathrm{J}}$ for the combination $\sigma_{Z}$ and $U_{K}$ and $r_{1}=0.967 r_{\mathrm{J}}$ for $\sigma_{F}$ and $U_{G}$. All $r_{1}$ values are listed in Table 2.

Green lines in Fig. 3 show $R m^{(1)}$ profiles for a typical convective velocity of $10 \mathrm{~cm} \mathrm{~s}^{-1}$ suggested by scaling laws (e.g., see Duarte et al. 2018). Numerical simulations show that the velocity increases with radius, an effect not taken into account here. The comparison of the different $R m^{(1)}$ profiles suggests that zonalflow-related dynamo action should dominate at least in the outer $9 \%$ in radius.

For the surface magnetic field of Jupiter we use the JRM09 model by Connerney et al. (2018), which provides information up to spherical harmonic degree $\ell=10$. The more recent model by Moore et al. (2018) is only slightly different. In order to check the impact of smaller-scale contributions, we also tested the numerical model G14 by Gastine et al. (2014), which reproduces the large-scale field of Jupiter and provides harmonics up to $\ell=426$. Since it turned out that the impact of the smaller scales is very marginal, the results are not shown here.

\section{Dissipative heating and entropy production in Jupiter}

Liu et al. (2008) constrain the depth of the zonal winds by assuming that the related total Ohmic heating should not exceed the heat flux out of the planet. Unfortunately, this assumption is 


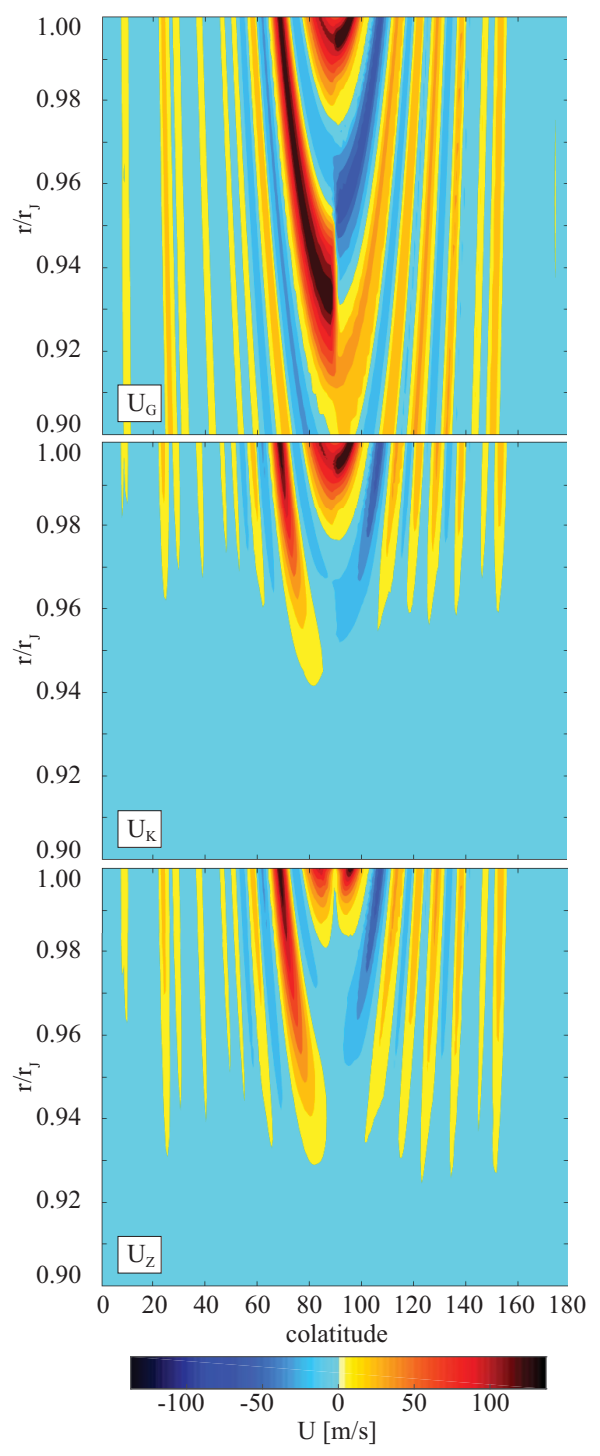

Fig. 2. Zonal flow models used in this study. Prograde flows are shown in red and yellow, while blue indicates retrograde directions.

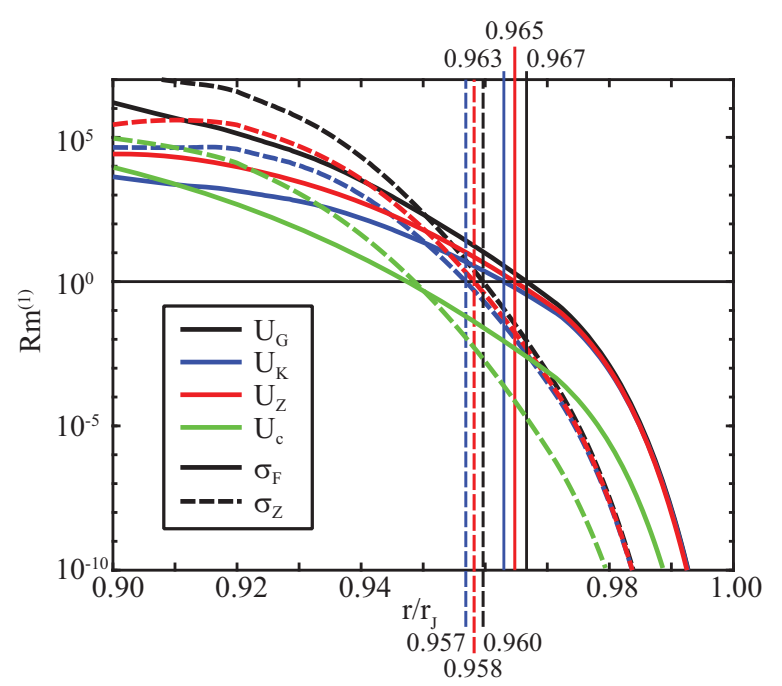

Fig. 3. Modified magnetic Reynolds numbers for the three zonal flow and the two conductivity models explored here. Vertical lines mark the radii $r_{1}$ where $R m^{(1)}=1$. Green lines show profiles for a typical convective velocity of $10 \mathrm{~cm} \mathrm{~s}^{-1}$ suggested by scaling laws. incorrect, as we show in the following. In order to arrive at more meaningful constraints, we start by reviewing some fundamental considerations.

In a quasi-stationary state, where flow and magnetic field are maintained by buoyancy and induction against dissipative losses, the conservation of energy simply states that the heat flux $Q_{\mathrm{o}}=Q\left(r_{\mathrm{o}}\right)$ through the outer boundary is the sum of the flux $Q_{\mathrm{i}}=Q\left(r_{\mathrm{i}}\right)$ through the inner boundary and the total internal heating $H$ :

$Q_{\mathrm{o}}=Q_{\mathrm{i}}+H$.

We note that neither viscous nor Ohmic heating contribute to $H$. Since flow and magnetic field are maintained by the heat flux through the system, they cannot be counted as net heat sources (Hewitt et al. 1975; Braginsky \& Roberts 1995).

When furthermore neglecting the effects of helium segregation, core erosion, and planetary shrinking as potential energy sources, the only remaining contribution is the slow secular cooling of the planet. The volumetric heat source is then given by

$h=\tilde{\rho} \tilde{T} \frac{\partial \tilde{S}}{\partial t}$,

where the tilde indicates the hydrostatic, adiabatic background state (Braginsky \& Roberts 1995). Assuming that convection maintains an adiabat at all times, $\partial \tilde{S} / \partial t$ remains homogeneous throughout the convective region and obeys (Jones 2014)

$\frac{\partial \tilde{S}}{\partial t}=\left(Q_{\mathrm{o}}-Q_{\mathrm{i}}\right) / \int_{V} \mathrm{~d} V \tilde{\rho} \tilde{T}$,

where $\int_{V} \mathrm{~d} V$ denotes an integration over the whole convective volume. We note however that the thermal evolution could be more complex should Jupiter indeed harbor stably stratified regions.

In order to assess dissipative heating, one must consider the local heat equation

$\tilde{\rho} \tilde{T}\left(\frac{\partial s}{\partial t}+\boldsymbol{U} \cdot \nabla s\right)=\nabla \cdot(k \nabla T)+h+\varphi$

where $\varphi$ denotes the volumetric dissipative heat source and $k$ the thermal conductivity. When assuming a steady state and adopting the anelastic approximation $\nabla \cdot(\tilde{\rho} \boldsymbol{U})=0$, the integration over the shell between the inner boundary $r_{i}$ and radius $r$ yields

$Q_{D}(r)+Q_{A}(r)=Q_{i}+\int_{r_{i}}^{r} \mathrm{~d} r^{\prime} \int_{F} \mathrm{~d} F\left(h+\varphi+\tilde{\rho} s U_{r} \frac{\partial \tilde{T}}{\partial r}\right)$.

The left-hand side is the total flux through the boundary at $r$, that is, the sum of the diffusive contribution,

$Q_{D}(r)=-\int_{F} \mathrm{~d} F k \frac{\partial T}{\partial r}$

and the advective contribution,

$Q_{A}(r)=\int_{F(r)} \mathrm{d} F \tilde{\rho} \tilde{T} s U_{r}$.

The right-hand side of Eq. (37) reflects the influx through the lower boundary $Q_{i}$ plus three volumetric contributions: the slow secular cooling, the dissipative heating, and the adiabatic cooling. Writing the adiabatic cooling in terms of $Q_{A}$ yields 
Table 2. Definition of the models (first two columns) and different radii: radius $r_{1}$ where $R m^{(1)}=1, r_{10}$ where $R m^{(1)}=10, r_{\Phi}$ where $\Phi_{O}=\Phi_{T}$, and $r_{\Theta}$ where $\Theta_{O}=\Theta_{T}$ (Cols. 3-6).

\begin{tabular}{ccccccccc}
\hline \hline Conduct. & Flow & $r_{1} / r_{\mathrm{J}}$ & $r_{10} / r_{\mathrm{J}}$ & $r_{\Phi} / r_{\mathrm{J}}$ & $r_{\Theta} / r_{\mathrm{J}}$ & $\left\langle\hat{B}_{r}\right\rangle /\left\langle\tilde{B}_{r}\right\rangle$ & $\Phi_{O} / \Phi_{T}$ & $\Theta_{O} / \Theta_{T}$ \\
\hline \multirow{2}{*}{$\sigma_{F}$} & $U_{G}$ & 0.967 & 0.960 & 0.961 & 0.955 & $2.8 \times 10^{-3}$ & $1.2 \times 10^{-1}$ & $2.3 \times 10^{-2}$ \\
& $U_{Z}$ & 0.965 & 0.957 & 0.956 & 0.948 & $2.2 \times 10^{-3}$ & $8.0 \times 10^{-2}$ & $1.5 \times 10^{-2}$ \\
& $U_{K}$ & 0.963 & 0.954 & 0.946 & 0.929 & $3.0 \times 10^{-3}$ & $4.8 \times 10^{-2}$ & $9.0 \times 10^{-3}$ \\
\hline \multirow{2}{*}{$\sigma_{Z}$} & $U_{G}$ & 0.960 & 0.956 & 0.956 & 0.953 & $1.8 \times 10^{-3}$ & $1.5 \times 10^{-1}$ & $2.5 \times 10^{-2}$ \\
& $U_{Z}$ & 0.958 & 0.954 & 0.953 & 0.949 & $1.6 \times 10^{-3}$ & $8.9 \times 10^{-2}$ & $1.5 \times 10^{-2}$ \\
& $U_{K}$ & 0.957 & 0.952 & 0.948 & 0.942 & $1.7 \times 10^{-3}$ & $2.9 \times 10^{-2}$ & $4.9 \times 10^{-3}$ \\
\hline
\end{tabular}

Notes. Column 7 provides the ratio of rms radial LIF to rms radial background field at $r_{1}$. Columns 8 and 9 give ratios $\Phi_{O} / \Phi_{T}$ and $\Theta_{O} / \Theta_{T}$ at $r_{1}$, respectively. Current density estimates $\boldsymbol{j}^{(O)}$ have been used.

the relation

$$
\begin{aligned}
Q_{D}(r)+Q_{A}(r)= & Q_{i}+\int_{r_{i}}^{r} \mathrm{~d} r^{\prime} \int_{F} \mathrm{~d} F(h+\varphi) \\
& -\int_{r_{i}}^{r} \mathrm{~d} r^{\prime} Q_{A}\left(r^{\prime}\right) / D_{T}\left(r^{\prime}\right),
\end{aligned}
$$

where $D_{T}=-\tilde{T} /(\partial \tilde{T} / \partial r)$, which is the thermal scale height.

Integrating Eq. (40) over the whole convective volume and using Eq. (33) reveals that the total dissipative heating $\Phi_{T}$ is balanced by the total adiabatic cooling:

$\Phi_{T}=\int_{V} \mathrm{~d} V \varphi=\int_{r_{i}}^{r_{o}} \mathrm{~d} r^{\prime} Q_{A}\left(r^{\prime}\right) / D_{T}\left(r^{\prime}\right)$.

The total adiabatic cooling is actually identical to the buoyancy power $P$ that drives convection and thus the dynamo mechanism. Multiplying the buoyancy term in the Navier-Stokes equation with velocity and integrating over the convective volume to yield the total convective power input indeed gives the same expression (Braginsky \& Roberts 1995). Equation (41) therefore simply states that dissipation is balanced by the power input $P$ to the system, a fact used in many scaling laws to establish how the rms magnetic field strength or the rms velocity scale with $P$ (Christensen \& Aubert 2006; Christensen et al. 2009; Davidson 2013; Yadav et al. 2013).

Equation (41) requires knowledge of $Q_{A}$ at each radius. Since $Q_{A}$ itself depends on the distribution of dissipative heat sources however, an additional condition is required. Assuming that Ohmic heating and adiabatic cooling not only cancel globally but, at least roughly, also at each radius offers a simple solution used in most scaling laws (though never stated explicitly). With the exception of thin thermal boundary layers, the heat flux is then dominated by the advective contribution, so that

$Q_{A}(r) \approx Q_{i}+\int_{r_{i}}^{r} \mathrm{~d} r \int_{F} \mathrm{~d} F h$.

Adopting the interior model by Nettelmann et al. (2012) and French et al. (2012) and the observed flux $Q_{\mathrm{o}}=3.35 \times 10^{17} \mathrm{~W}$ from the interior of the planet (Guillot \& Gautier 2015) allows us to calculate $h$ via Eq. (34). Because the inner core occupies only $10 \%$ in radius, $Q_{i}$ can be neglected. When for example assuming that $h$ also describes the cooling of the rocky core, $Q_{\mathrm{i}}$ is two orders of magnitude smaller than $Q_{\mathrm{o}}$.
Plugging Eq. (42) into Eq. (41) finally allows us to calculate the total dissipative heating:

$\Phi_{T}=1.20 \times 10^{18} \mathrm{~W}$.

The result reveals that dissipative heating can in fact exceed the heat flux out of the interior of Jupiter by a factor of 3.6. Gastine et al. (2014) obtained a power estimate that is about $50 \%$ smaller than this latter figure because they used a simplified formula provided by Christensen et al. (2009).

Considering the entropy rather than the heat balance avoids the need to come up with an additional condition (Hewitt et al. 1975; Gubbins et al. 1979, 2003; Braginsky \& Roberts 1995). Dividing the heat equation Eq. (36) by temperature and integrating over the convective volume yields the entropy budget:

$\frac{Q_{o}}{\tilde{T}_{o}}=\frac{Q_{i}}{\tilde{T}_{i}}+\int_{V} \mathrm{~d} V\left(\frac{h}{\tilde{T}}+k\left|\frac{\partial T / \partial r}{\tilde{T}}\right|^{2}+\frac{\varphi}{\tilde{T}}\right)$,

where we have once more used the anelastic approximation $\nabla \cdot(\tilde{\rho} \boldsymbol{U})=0$. When assuming that the temperature profile stays close to the adiabat, the total dissipative entropy production $\Theta$ can thus be approximated by

$\Theta_{T}=\int_{V} \mathrm{~d} V \frac{\varphi}{\tilde{T}} \approx \frac{Q_{\mathrm{o}}}{\tilde{T}_{\mathrm{o}}}-\frac{Q_{\mathrm{i}}}{\tilde{T}_{\mathrm{i}}}-\int_{V} \mathrm{~d} V \frac{h}{\tilde{T}}-\int_{V} \mathrm{~d} V k\left|\frac{\partial \tilde{T} / \partial r}{\tilde{T}}\right|^{2}$.

An upper bound for the total dissipative heating can be derived when assuming that $\tilde{T}_{i}$ is the highest temperature in the system (Hewitt et al. 1975; Currie \& Browning 2017):

$\Phi<\tilde{T}_{i} \int_{V} \mathrm{~d} V \frac{\varphi}{\tilde{T}}<\frac{\tilde{T}_{i}}{\tilde{T}_{o}} Q_{\mathrm{o}}$.

Using once more the internal model by Nettelmann et al. (2012) puts the upper bound at $10^{2} Q_{\mathrm{o}}$ for Jupiter, which is at least consistent with estimate (43).

When complementing the internal model with the thermal conductivity profile by French et al. (2012), we can quantify the different terms in the entropy budget of Jupiter (Eq. (45)). Because of the strong temperature contrast between the outer boundary and the deeper convective region, the entropy flux through the outer boundary clearly dominates. The total dissipative entropy production is therefore given by

$\Theta_{T} \approx Q_{\mathrm{o}} / \tilde{T}_{\mathrm{o}}=2.0 \times 10^{15} \mathrm{~W} / \mathrm{K}$. 
The second-largest term in Eq. (45), the entropy due to the secular cooling, is already two orders of magnitude smaller, at $3.0 \times 10^{13} \mathrm{~W} / \mathrm{K}$. The two remaining terms, entropy flux through the inner boundary and the diffusive entropy flux down the adiabat, are only of order $10^{11} \mathrm{~W} / \mathrm{K}$.

Since the magnetic diffusivity is about $10^{6}$ times larger than its viscous counterpart in planetary dynamo regions, Ohmic heating is by far the dominating factor. We can use the current density estimates to predict the Ohmic heating due to the zonal flows above radius $r$ :

$\Phi_{O}(r)=\int_{r}^{r_{J}} \mathrm{~d} r^{\prime} \int_{F} \mathrm{~d} F \frac{\boldsymbol{j}^{2}}{\sigma}$.

The conditions

$\Phi_{O}(r) \leq \Phi_{T}=1.20 \times 10^{18} \mathrm{~W}$

provides a possible constraint for the depth of the zonal winds in Jupiter.

The dissipative entropy production related to the Ohmic heating is given by

$\Theta_{O}(r) \approx \int_{r}^{r_{J}} \mathrm{~d} r^{\prime} \int_{F} \mathrm{~d} F \frac{j^{2}}{\sigma \tilde{T}}$.

This can be used for the alternative depth constraint

$\Theta_{O}(r) \leq \Theta_{T}=2.0 \times 10^{15} \mathrm{~W} / \mathrm{K}$.

\section{Dynamo action in the SDCR of Jupiter}

In the following we apply the methods developed above to the SDCR of Jupiter. We first estimate the electric currents and induced fields in Sect. 4.1 and then explore whether Ohmic heating or entropy can serve to constrain the depth of the zonal winds in Sect. 4.2.

\subsection{Electric currents and locally induced field}

We start by discussing the current estimates for the different zonal flow and conductivity model combinations. Figure $4 \mathrm{a}$ compares rms values of integral estimates $j^{(I)}$ and Ohm's law estimates $\boldsymbol{j}^{(O)}$ for conductivity model $\sigma_{F}$ and flow $U_{Z}$. The integral estimates of the latitudinal currents are at least 40 times larger than their azimuthal counterparts for all conductivity and flow model combinations. When using $\boldsymbol{j}^{(O)}$ as outer boundary condition for $\boldsymbol{j}^{(I)}$ (blue line in Fig. 4), both estimates remain very similar down to about $0.98 r_{\mathrm{J}}$. At $0.97 r_{\mathrm{J}}$ however, $j^{(I)}$ is already about $50 \%$ larger than $j^{(O)}$, and at $0.96 r_{\mathrm{J}}$ the difference has increased to about $250 \%$. When assuming a vanishing outer boundary current for $j^{(I)}$, the differences are even larger: $\boldsymbol{j}^{(I)}$ (green line) is 3.5 times larger than $\boldsymbol{j}^{(O)}$ at $0.97 r_{\mathrm{J}}$ and about 6 times larger at $0.96 r_{\mathrm{J}}$.

Estimates of the rms horizontal and radial LIF are shown in Fig. 4b, based on $\boldsymbol{j}^{(O)}$ and Eq. (20) for the horizontal and on Eq. (26) for the radial components. The radial LIF is between two and three orders of magnitude smaller than its horizontal counterpart. The rougher estimates (Eqs. (23) and (28)), based on $R m^{(1)}$ and $R m^{(2)}$, respectively, provide values that are less than a factor two smaller and can thus safely be used for order-of-magnitude assessments; they correctly predict that the rms azimuthal LIF reaches the level of the background field at $r_{1}$ and also that the ratio of radial to azimuthal LIF is about
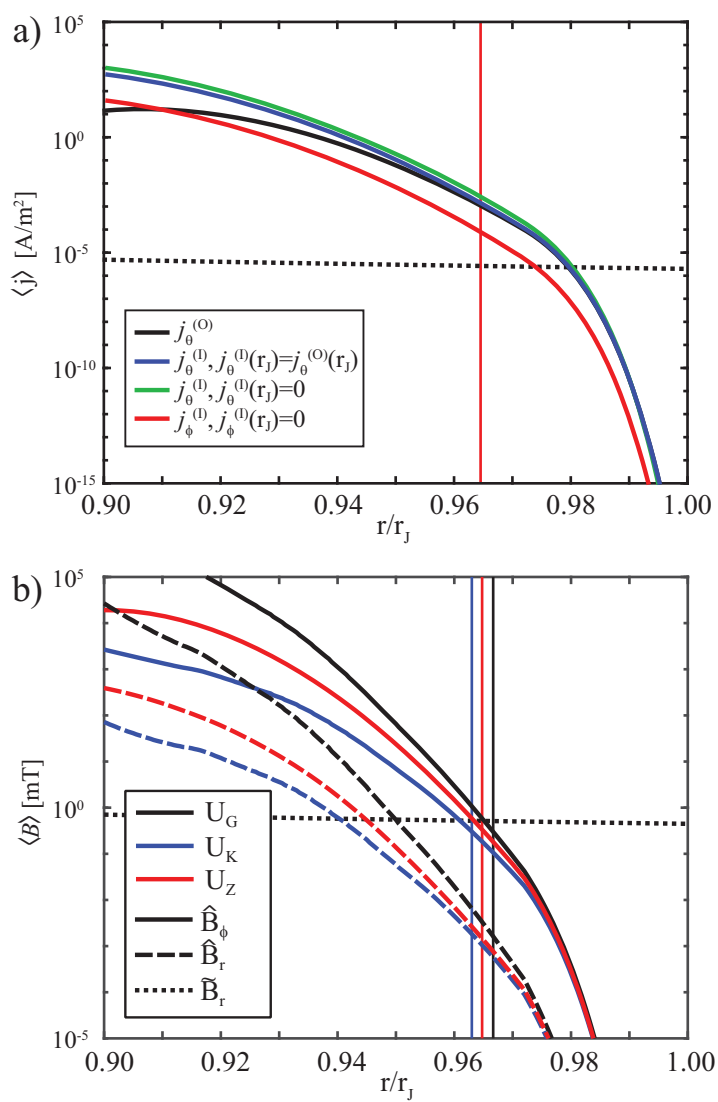

Fig. 4. Panel $a$ : rms current density estimates for flow model $U_{Z}$ and conductivity model $\sigma_{F}$. Panel $b$ : estimates of rms radial and horizontal LIF. The profile of $\left\langle\tilde{B}_{r}\right\rangle$ has been included for comparison. Vertical lines mark the radii where $R m^{(1)}=1$ for the different flow models. Current density estimates $\boldsymbol{j}^{(O)}$ and conductivity model $\sigma_{F}$ have been used.

$R m^{(2)} / R m^{(1)}=D_{\lambda} / r_{\mathrm{J}}$. At $r_{1}$, the rms radial LIF is thus roughly three orders of magnitude smaller than the background field or the horizontal LIF. Table 2 lists the relative rms radial LIF (Col. 7) at $r_{1}$ (Col. 3) for all $\sigma$ and flow combinations when using $j^{(O)}$.

Wicht et al. (2019) demonstrate that the estimates based on Ohm's-law not only provide good rms but also good local values for their Jupiter-like dynamo simulations. Figure 5 shows the radial surface field of JRM09 in panel a and the radial LIF for $\sigma_{F}$ and $U_{Z}$ at $r_{1}$ in panel b. A very distinct pattern of localized field patches can be found where the fast zonal jets around the equator interact with the strong blue patch in the JRM09 model.

The zonal flow pattern remains recognizable in the LIF, as is clearly demonstrated in Fig. 6, which compares zonal flow profiles in panel a with the azimuthal rms of the radial LIF in panel b. Due to the flow geometry, the currents and LIF show a depth-dependent phase shift relative to the surface jets. The equatorial jet, which is so prominent at the surface, contributes very little to dynamo action, since it does not reach down to depths where the electrical conductivity is more significant.

Figure 7 compares spherical harmonic power spectra of the background radial field and the radial LIF. As already apparent from the map shown in Fig. 5, the LIF is dominated by smallerscale contributions. The spherical harmonic degree spectrum results from the convolution of the complex latitudinal zonal flow structure with the background field. At $r_{1}$, the dipole contribution in the LIF is about $10^{-4}$ times smaller than the respective background field contribution. For degree $\ell=10$, the ratio has 

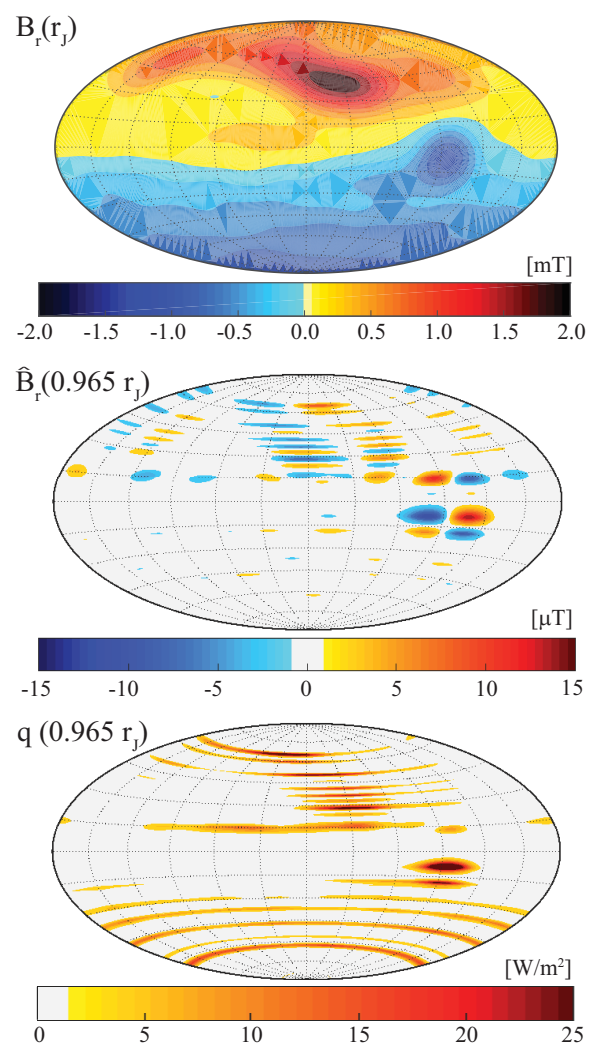

Fig. 5. Maps of panel $a$ : radial surface field in the Jupiter field model JRM09, panel $b$ : radial LIF at $r_{1}=0.965 r_{\mathrm{J}}$, and panel $c$ : local Ohmic heating $q=\int_{r}^{r_{\mathrm{J}}} \mathrm{d} r \boldsymbol{j}^{2} / \sigma$ at $r=r_{1}=0.965 r_{\mathrm{J}}$. Flow model $U_{Z}$, conductivity $\sigma_{F}$, and current density estimates $\boldsymbol{j}^{(O)}$ (for panel $c$ ) have been used. Outward (inward) directed field is shown in red (blue).

increased to $10^{-2}$. The spectrum peaks at $\ell=12$ but has also significant contributions from even higher degrees.

The spherical harmonic order spectrum, shown in panel $b$ of Fig. 7, is very different. The action of the axisymmetric zonal flow on $\tilde{B}_{r}$ excites no additional harmonic orders so that the spectrum remains confined to $m \leq 10$. The LIF spectrum is rather flat but has no axisymmetric contribution. At $m=10$, the rms LIF amplitude reaches roughly $25 \%$ of the background field.

The results for the conductivity model $\sigma_{F}$ presented so far can roughly be scaled to model $\sigma_{Z}$ by multiplying with the conductivity ratio $\sigma_{Z} / \sigma_{F}$. Around $0.97 r_{\mathrm{J}}$, the LIF is two orders of magnitude weaker, and the difference decreases with depth, reaching about one order of magnitude around $0.96 r_{\mathrm{J}}$. Where $R m^{(1)}=1$, on the other hand, the LIF reaches comparable values for both conductivity models. The different flow models yield very similar LIF patterns, albeit with the different amplitudes indicated in Fig. 4.

\subsection{Ohmic heating and entropy constraint}

We now use the electric current estimates to calculate Ohmic heating and entropy production. Panel c of Fig. 5 shows the map of Ohmic heat flux density $q=\int_{r}^{r_{J}} \mathrm{~d} r \boldsymbol{j}^{2} / \sigma$ at radius $r_{1}$ when using $\boldsymbol{j}^{(O)}, \sigma_{F}$, and $U_{Z}$. The currents induced by interaction between the fierce zonal jets close to the equator and the strong blue patch in JRM09 not only yield a highly localized LIF but also intense local heating. While the action of various other zonal jets reaches a lower level, the related pattern remains roughly recognizable in the form of thin heating bands. The azimuthal mean

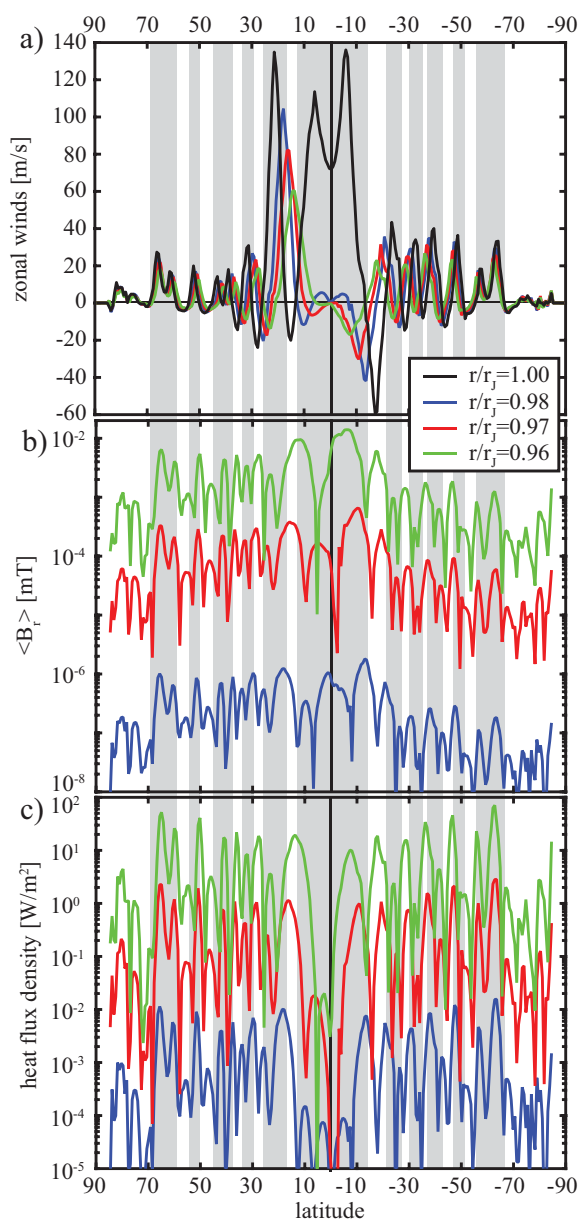

Fig. 6. Panel $a$ : $U_{Z}$ profiles at different depths; the grey background color highlights where surface jets are prograde. Panel $b$ : azimuthal rms radial LIF and panel $c$ : heat flux $q=\int_{r}^{r_{J}} \mathrm{~d} r j^{2} / \sigma$ at three different depths. Conductivity model $\sigma_{F}$, flow $U_{Z}$, and estimate $j^{(O)}$ have been used.

of $q$ shown in panel c of Fig. 6 clearly illustrates the correlation between heating and the zonal jets. Like for the LIF, there is a depth-dependent phase shift between the observed surface zonal wind profile and the Ohmic heating pattern.

Panel a of Fig. 8 compares the Ohmic heating profiles $\Phi_{O}(r)$ for the different zonal flow and electrical conductivity models. Because of the extremely low conductivity, heating remains negligible in the outer two percent in radius. When using $j^{(O)}$, the outermost radius where $\Phi_{O}$ reaches the level of $\Phi_{T}$ is $r_{\Phi}=0.950 r_{\mathrm{J}}$ for flow $U_{G}$ and both conductivity models. When using $U_{K}$ and $\sigma_{F}$, the Ohmic heating always remains below $\Phi_{T}$. Results based on $\boldsymbol{j}^{(I)}$ (not shown) are less sensitive to the differences between the three flow models at depth and are generally similar to the results for $U_{G}$ and $j^{(O)}$.

The different $r_{\Phi}$ values where $\Phi_{O}=\Phi_{T}$ have been marked by vertical lines in Fig. 8 and are listed in Col. 5 of Table 2 . All are located below the radii $r_{1}$ where $R m^{(1)}=1$ for the respective model combinations (Col. 3) and thus in a region where the approximations employed here break down. The maximum Ohmic heating reached at $r_{1}$ remains nearly one order of magnitude below $\Phi_{T}$ (see Col. 8 of Table 2).

Similar inferences hold for the entropy production shown in panel $b$ of Fig. 8. The entropy condition is less strict than the power-based heat condition, and the radii $r_{\Theta}$ where the different models exceed the threshold $\Theta_{T}$ (Col. 6 of Table 2) are somewhat 

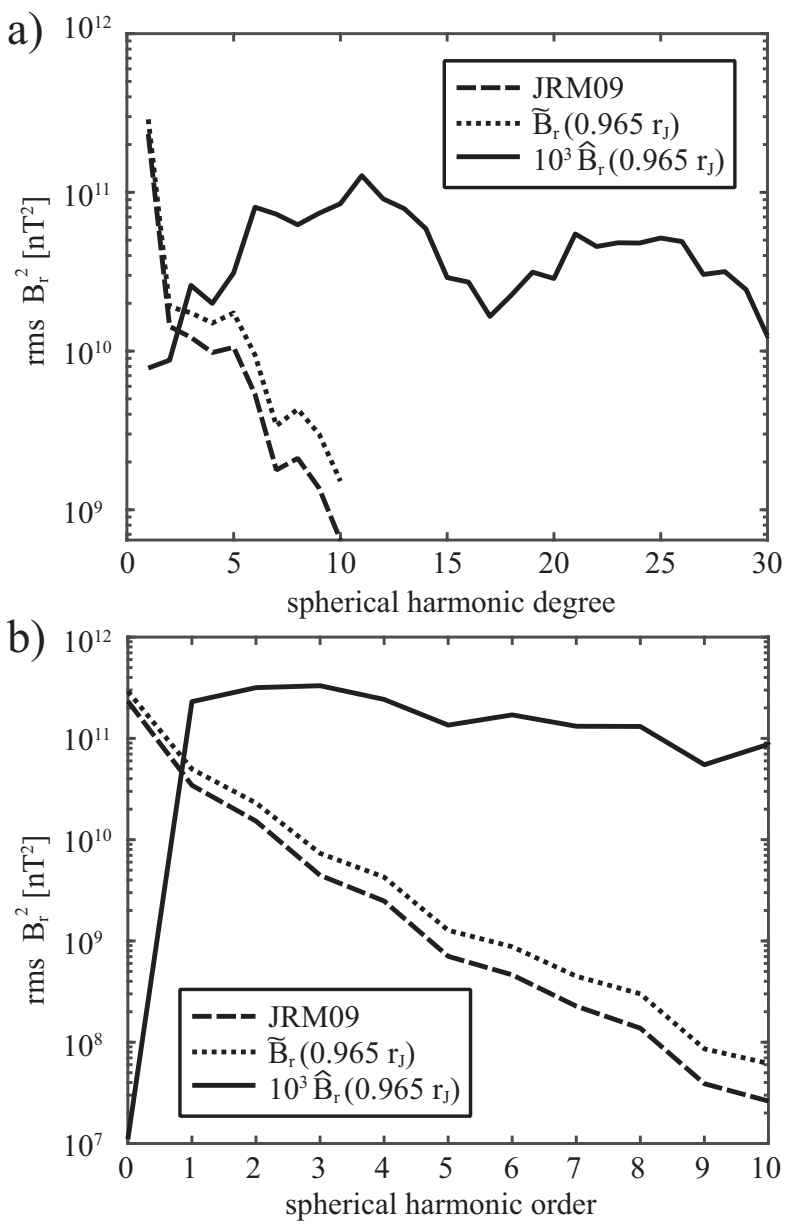

Fig. 7. Power spectra of rms radial field contributions (MauersbergerLowes) for JRM09, the downward continued $\tilde{B}_{r}$ and the radial LIF $\hat{B}_{r}$ at $r_{1}=0.965 r_{\mathrm{J}}$ : panel $a$ : spherical harmonic degree spectrum; panel $b$ : harmonic order spectrum. The LIF has been amplified by 1000. Flow $U_{Z}$ and conductivity $\sigma_{F}$ have been used.

deeper than respective $r_{\Phi}$ values. The largest value of $r_{\Theta}=0.955$ is found for the combination $\mathrm{U}_{G}$ and $\sigma_{Z}$. The combination of $U_{K}$ and $\sigma_{F}$, on the other hand, yields the deepest value of $r_{\Theta}=$ 0.929 .

The exploration of numerical dynamo simulations by Wicht et al. (2019) suggest that $j^{(O)}$ may provide an acceptable estimate for a limited region below $r_{1}$, at least down to where $R m^{(1)}=5$. Column 4 of Table 2 demonstrates that even the radius $r_{10}$ where $R m^{(1)}=10$ lies deeper than $r_{\Phi}$ for most flow and conductivity combinations. The only exceptions are the results for the geostrophic flow. This could indicated that strictly geostrophic flows would indeed violate the heating contraint.

\section{Discussion and conclusion}

The dominance of Ohmic dissipation in the outer few percent of the radius of Jupiter leads to simple quasi-stationary dynamo action. This can be exploited for estimating the electric currents and the LIFs with surprisingly high quality (Wicht et al. 2019), once a conductivity profile, a surface magnetic field model, and flow model are given. Here we explored two conductivity profiles, used the new Juno-based JRM09 field model, and tested two zonal flow models suggested from inversions of Juno gravity measurements. A geostrophic zonal flow model was also considered as a third option.
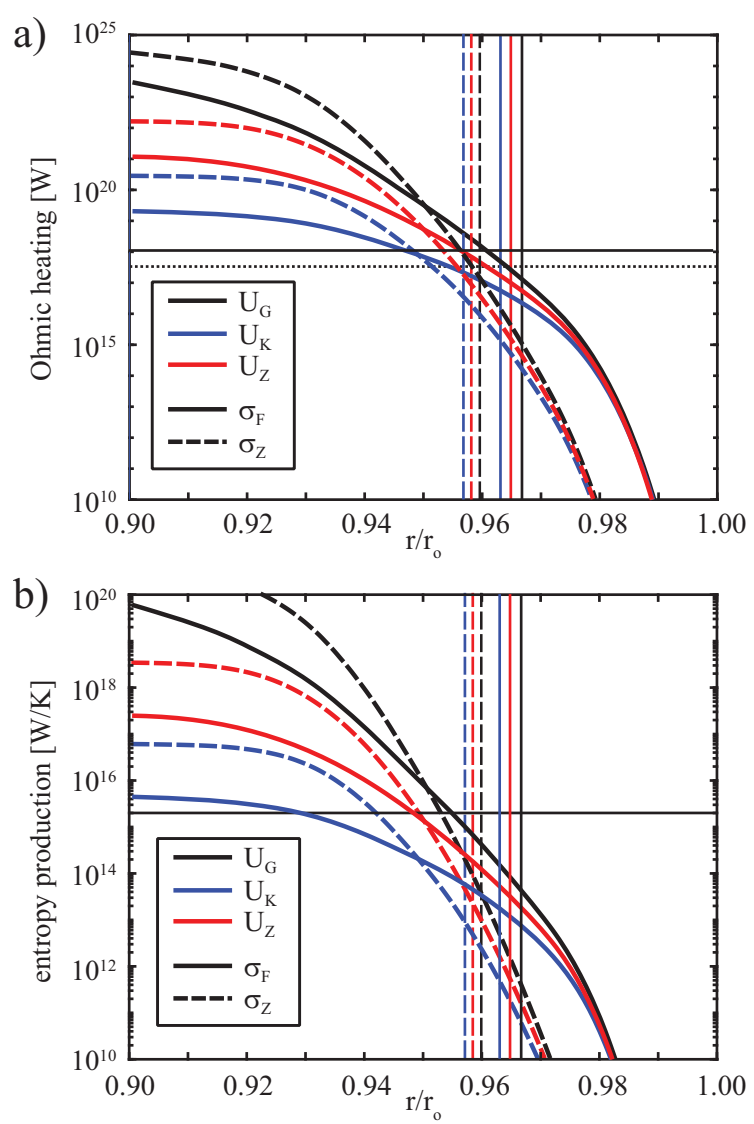

Fig. 8. Profiles of panel $a$ : Ohmic heating and panel $b$ : entropy production in the layer above radius $r$ for current estimate $j^{(O)}$. In $(a)$ the solid horizontal line shows the total convective power of $1.2 \times 10^{18} \mathrm{~W}$, while the dotted horizontal line shows the heat flow of $Q_{\mathrm{o}}=3.35 \times 10^{17} \mathrm{~W}$ from the interior of Jupiter. In $(b)$ the horizontal line indicates the total dissipative entropy production predicted by the entropy flux $\Theta_{T}=$ $Q_{\mathrm{o}} / T_{\mathrm{o}}=2.0 \times 10^{15} \mathrm{~W} / \mathrm{K}$ through the outer boundary. Vertical lines mark the radii $r_{1}$ where $\mathrm{Rm}^{(1)}=1$ (see Fig. 3).

The estimates roughly apply to the upper four percent in radius, or roughly $3000 \mathrm{~km}$, where the modified magnetic Reynolds number $R m^{(1)}$ is smaller than one. The radial LIF in this quasi-stationary dynamo region typically reaches rms values in the order of $\mu \mathrm{T}$ with peak values up to $15 \mu \mathrm{T}$. Could such a small contribution be measured by the Juno magnetometer? The instrument has been designed to provide a nominal vector accuracy of 1 in $10^{4}$. Since the surface field reaches peak values of about $2 \mathrm{mT}$, the LIF could indeed be detectable.

One would still have to separate the LIF from contributions produced deeper in the planet. What should help with this task is the distinct pattern imprinted by the zonal flows, which also leads to a distinct magnetic spectrum. The LIF spectrum peaks at degree $\ell=12$ and has significant contributions at even higher degrees. At $\ell=10$, the largest degree provided by JRM09, the LIF amounts to about $1 \%$ of the background field, which seems smaller than the estimated JRM09 precision (Connerney et al. 2018). Updated future models, based on a larger number of Juno orbits, will provide smaller-scale details and increase the chances of identifying the LIF. Another possibility is a dedicated analysis of measurements around the "big blue spot" in JRM09, where induction effects are particularly strong.

Our analysis of the heat balance of Jupiter shows that Ohmic heating can significantly exceed the heat flux $Q_{\mathrm{o}}$ out of the interior of the planet. Using the interior model by 
Nettelmann et al. (2012) and French et al. (2012) suggests a total dissipative heating of $\Phi_{T}=3.58 Q_{\mathrm{o}}=1.20 \times 10^{18} \mathrm{~W}$.

It would be interesting to repeat this assessment for the newer Jupiter models that include stably stratified regions (Debras \& Chabrier 2019). However, the most important constraint is the knowledge of $Q_{\mathrm{o}}$, and the somewhat different distribution of internal heat sources implied by the newer models can only have a limited effect.

While the total Ohmic heating remains typically one order of magnitude below $\Phi_{T}$, we find extreme lateral variations. Peak values in the Ohmic heating density reach $25 \mathrm{~W} \mathrm{~m}^{-2}$ around the "blue spot" in the JRM09, which is nearly five times larger than the mean heat flux density from the interior of Jupiter. These peak values are reached at the bottom of the quasi-stationary region, that is, at a depth of $3000 \mathrm{~km}$. This is much deeper than any (current) remote instrument could reach. For example MWR, the micro-wave instrument on Juno, hopes to detect temperature radiation from up to $1 \mathrm{kbar}$, which corresponds to a depth of about $600 \mathrm{~km}$. However, the local heating may trigger convective plumes that rise to shallower depths and thus become detectable.

We also estimated the entropy flux out of the interior of Jupiter to $2.0 \times 10^{15} \mathrm{~W} \mathrm{~K}^{-1}$. The entropy produced by zonalwind-related Ohmic heating in the quasi-stationary region does not exceed this value for any model combination. This means that neither Ohmic heating nor the entropy production offer any reliable constraint on the depth of the zonal winds.

Below the quasi-stationary region, electric fields become a significant contribution to Ohm's law, tend to oppose induction effects, and lead to weaker electric currents than predicted by our approximations. Wicht et al. (2019) demonstrate that the currents remain roughly constant below the depth where $R m^{(1)} \approx 5$ in their numerical simulations. However, this may be different in Jupiter where the magnetic Reynolds numbers reach values orders of magnitude higher than in their computer models.

Figure 3 demonstrates that $R m^{(1)}$ increases to a value of at least $10^{3}$ at $0.90 r_{\mathrm{J}}$. This is a consequence of the electrical conductivity profiles that easily overcompensate the depth-decrease in zonal flow velocities indicated by Juno gravity measurements. The zonal flows may therefore actually play a larger role for dynamo action below the quasi-stationary region. While the gravity data convincingly show that the zonal winds must be significantly weaker below about $0.96 r_{\mathrm{J}}$, they cannot uniquely constrain their structure or amplitude at this depth.

It has been speculated that the fast observed zonal winds may remain confined to a thin weather layer, where differential solar heating and also moist convection could significantly contribute to the dynamics (see e.g., Showman 2007 or Thomson \& McIntyre 2016). Kong et al. (2018) show that the gravity signal can then be explained by an independent zonal flow system that reaches down to about $0.7 r_{\mathrm{J}}$ with typical amplitudes of about $1 \mathrm{~m} \mathrm{~s}^{-1}$ and that has larger latitudinal scales than the surface winds. The strongest local dynamo action happens towards the bottom of the quasi-stationary region where models $U_{K}$ and $U_{Z}$ reach velocities of about $10 \mathrm{~m} \mathrm{~s}^{-1}$. The currents and magnetic fields induced by this alternative flow model should therefore be roughly an order of magnitude weaker than for $U_{K}$ or $U_{Z}$. Consequently, Ohmic heating and entropy production would be two orders of magnitude lower and play practically no role for the global power or entropy budgets.

Below $0.96 r_{\mathrm{J}}$, full 3D numerical simulations would be required to model the zonal-wind-related dynamo action. However, since they cannot be run at altogether realistic parameters and generally yield a much simpler zonal wind pattern, the results must be interpreted with care (Gastine et al. 2014;
Jones 2014; Duarte et al. 2018; Dietrich \& Jones 2018). These simulations suggest that even the weaker zonal winds at depth would significantly shear the large-scale field produced by the deeper primary dynamo action. The resulting strong longitudinal (toroidal) flux bundles are converted into observable radial field by the small-scale convective flows present in this region. The combined action of primary and secondary dynamo typically yields a radial surface field that is characterized by longitudinal banded structures and large-scale patches with wavenumber one or two, resulting in a morphology that is often reminiscent of the recent Juno-based field model JRM09 (Gastine et al. 2014; Duarte et al. 2018; Dietrich \& Jones 2018).

Acknowledgements. This work was supported by the German Research Foundation (DFG) in the framework of the special priority programs "PlanetMag" (SPP 1488) and "Exploring the Diversity of Extrasolar Planets" (SPP 1992).

\section{References}

Braginsky, S., \& Roberts, P. 1995, Geophys. Astrophys. Fluid Dyn., 79, 1 Cao, H., \& Stevenson, D. J. 2017, Icarus, 296, 59

Christensen, U., \& Aubert, J. 2006, Geophys. J. Int., 116, 97

Christensen, U. R., Holzwarth, V., \& Reiners, A. 2009, Nature, 457, 167

Connerney, J. E. P., Kotsiaros, S., Oliversen, R. J., et al. 2018, Geophys. Res. Lett., 45, 2590

Currie, L. K., \& Browning, M. K. 2017, ApJ, 845, L17

Davidson, P. A. 2013, Geophys. J. Int., 195, 67

Debras, F., \& Chabrier, G. 2019, ApJ, 872, 100

Dietrich, W., \& Jones, C. A. 2018, Icarus, 305, 15

Duarte, L. D. V., Wicht, J., \& Gastine, T. 2018, Icarus, 299, 206

French, M., Becker, A., Lorenzen, W., et al. 2012, ApJS, 202, 5

Galanti, E., Kaspi, Y., Miguel, Y., et al. 2019, Geophys. Res. Lett., 46, 616

Gastine, T., Wicht, J., Duarte, L. D. V., Heimpel, M., \& Becker, A. 2014, Geophys. Res. Lett., 41, 5410

Gubbins, D., Masters, T. G., \& Jacobs, J. A. 1979, Geophys. J., 59, 57

Gubbins, D., Alfè, D., Masters, G., Price, G., \& Gillan, M. 2003, Geophys. J. Int., 155,609

Guillot, T., \& Gautier, D. 2015, in Treatise on Geophysics, Planets and Moons, 2nd edn, ed. T. Spohn (Amsterdam: Elsevier), 10, 439

Guillot, T., Miguel, Y., Militzer, B., et al. 2018, Nature, 555, 227

Heimpel, M., Gastine, T., \& Wicht, J. 2016, Nat. Geosci., 9, 19

Hewitt, J. M., McKenzie, D. P., \& Weiss, N. O. 1975, J. Fluid Mech., 68, 721

Iess, L., Folkner, W. M., Durante, D., et al. 2018, Nature, 555, 220

Iess, L., Militzer, B., Kaspi, Y., et al. 2019, Science, 364, aat2965

Jones, C. A. 2014, Icarus, 241, 148

Kaspi, Y., Galanti, E., Hubbard, W. B., et al. 2018, Nature, 555, 223

Knudson, M. D., Desjarlais, M. P., Preising, M., \& Redmer, R. 2018, Phys. Rev. B, 98, 174110

Kong, D., Zhang, K., Schubert, G., \& Anderson, J. D. 2018, Proc. Natl. Acad. Sci., 115, 8499

Liu, J., Goldreich, P. M., \& Stevenson, D. J. 2008, Icarus, 196, 653

Militzer, B., Soubiran, F., Wahl, S. M., \& Hubbard, W. 2016, J. Geophys. Res. (Planets), 121, 1552

Moore, K. M., Yadav, R. K., Kulowski, L., et al. 2018, Nature, 561, 76

Moore, K. M., Cao, H., Bloxham, J., et al. 2019, Nat. Astron., 3, 730

Nellis, W. J., Weir, S. T., \& Mitchell, A. C. 1996, Science, 273, 936

Nellis, W. J., Weir, S. T., \& Mitchell, A. C. 1999, Phys. Rev. B, 59, 3434

Nettelmann, N., Becker, A., Holst, B., \& Redmer, R. 2012, ApJ, 750, 52

Porco, C. C., West, R. A., McEwen, A., et al. 2003, Science, 299, 1541

Ridley, V. A., \& Holme, R. 2016, J. Geophys. Res., 121, 309

Schöttler, M., \& Redmer, R. 2018, Phys. Rev. Lett., 120, 115703

Showman, A. P. 2007, J. Atm. Sci., 64, 3132

Thomson, S. I., \& McIntyre, M. E. 2016, J. Atm. Sci., 73, 1119

Vasavada, A. R., \& Showman, A. P. 2005, Rep. Prog. Phys., 68, 1935

Weir, S. T., Mitchell, A. C., \& Nellis, W. J. 1996, Phys. Rev. Lett., 76, 1860

Wicht, J., French, M., Stellmach, S., et al. 2018, in Magnetic Fields in the Solar System, eds. H. Lühr, J. Wicht, S. A. Gilder, \& M. Holschneider (Cham, Switzerland: Springer), 7

Wicht, J., Gastine, T., \& Duarte, L. D. V. 2019, J. Geophys. Res. Planets, 124, 837

Yadav, R. K., Gastine, T., Christensen, U. R., \& Duarte, L. D. V. 2013, ApJ, 774,6

Zaghoo, M., \& Collins, G. W. 2018, ApJ, 862, 19 